\title{
Application of the CHNS-Analysis for Studying of Polyoxometallates and Ionic Liquids in the Catalytic Systems
}

\author{
Olga S. Koscheeva ${ }^{a}$, \\ Lidia I. Kuznetsova*b and Nina I. Kuznetsova ${ }^{\text {b }}$ \\ ${ }^{a}$ Nikolaev Institute of Inorganic Chemistry SB RAS \\ Novosibirsk, Russian Federation \\ ${ }^{b}$ Boreskov Institute of Catalysis SB RAS \\ Novosibirsk, Russian Federation
}

Received 10.03.2020, received in revised form 15.04.2020, accepted 01.05.2020

\begin{abstract}
The method of CHNS-analysis in combination with ${ }^{31} \mathrm{P}$ NMR and UV-VIZ spectroscopy was used to study polyoxometallates and ionic liquids as components of liquid-phase catalytic systems. The composition of imidazolium and TBA salts of tungstophosphate anions active in the olefin oxidation with $\mathrm{H}_{2} \mathrm{O}_{2}$ was determined. A method for quantification of [BMIm] Br, $\mathrm{Bu}_{4} \mathrm{NBr}, \mathrm{Bu}_{4} \mathrm{NHSO}_{4}$ in solutions using the CHNS-analysis is proposed. The capabilities of the technique with applying ionic liquids to an inert porous support are evaluated. Using the obtained results, the effect of ionic liquids in combination with a Pt/C catalyst and $\mathrm{H}_{3} \mathrm{PMo}_{12} \mathrm{O}_{40}$ heteropolyacid in the oxidation of cyclohexane with $\mathrm{O}_{2}-\mathrm{H}_{2}$ gas mixture was determined.
\end{abstract}

Keywords: imidazolium, tetrabutylammonium cations, tungstophosphate anions, peroxo complexes, ionic liquids, CHNS-analysis, ${ }^{31}$ P NMR.

Citation: Koscheeva O.S., Kuznetsova L.I., Kuznetsova N.I. Application of the CHNS-analysis for studying of polyoxometallates and ionic liquids in the catalytic systems, J. Sib. Fed. Univ. Chem., 2020, 13(2), 221-231. DOI: 10.17516/1998-2836-0177

(C) Siberian Federal University. All rights reserved

This work is licensed under a Creative Commons Attribution-NonCommercial 4.0 International License (CC BY-NC 4.0).

* Corresponding author E-mail address: livkuzn@catalysis.ru 


\title{
Применение метода CHNS-анализа
}

\section{для изучения полиоксометаллатов \\ и ионных жидкостей в каталитических системах}

\author{
О.С. Кощеева ${ }^{\text {a }, ~ Л . И . ~ К у з н е ц о в а ~}{ }^{\tilde{\sigma}}$, Н.И. Кузнецова ${ }^{\mathbf{0}}$ \\ ${ }^{a}$ Институт неорганической химии \\ им. А.В. Николаева СО РАН \\ Российская Федерация, Новосибирск \\ ${ }^{6}$ Институт катализа им. Г.К. Борескова СО РАН \\ Российская Федерачия, Новосибирск
}

Аннотация. Метод CHNS-анализа в сочетании с ЯMР ${ }^{31} \mathrm{P}$ и УФ-ВИД-спектроскопией применен для изучения полиоксометаллатов и ионных жидкостей как компонентов жидкофазных каталитических систем. Определен состав активных в окислении олефинов $\mathrm{H}_{2} \mathrm{O}_{2}$ имидазолиевых и ТВА-солей вольфрамофосфат-анионов. Предложен способ определения малых концентраций [BMIm] $\mathrm{Br}, \mathrm{Bu}_{4} \mathrm{NBr}, \mathrm{Bu}_{4} \mathrm{NHSO}_{4}$ в растворах с помощью CHNS-анализа. Оценены возможности методики с нанесением ионных жидкостей на инертный пористый носитель. С использованием полученных результатов определено действие ионных жидкостей в сочетании с катализатором $\mathrm{Pt} / \mathrm{C}$ и гетерополикислоты $\mathrm{H}_{3} \mathrm{PMo}_{12} \mathrm{O}_{40}$ в окислении циклогексана газовой смесью $\mathrm{O}_{2}-\mathrm{H}_{2}$.

Ключевые слова: имидазолий, тетрабутиламмоний катионы, вольфрамофосфат анионы, пероксокомплексы, ионные жидкости, CHNS-анализ, ЯMP ${ }^{31} \mathrm{P}$.

Цитирование: Кощеева, O.C. Применение метода CHNS-анализа для изучения полиоксометаллатов и ионных жидкостей в каталитических системах / О.С. Кощеева, Л.И. Кузнецова, Н.И. Кузнецова // Журн. Сиб. федер. ун-та. Химия, 2020. 13(2). С. 221-231. DOI: 10.17516/1998-2836-0177

\section{Введение}

Полиоксометаллаты (ПОМ), так же как и ионные жидкости (ИЖ), могут состоять из алкилзамещенных катионов аммония, пиридиния, имидазолия и неорганических анионов. Благодаря такой природе ПОМ и ИЖ могут растворяться в органических средах и служить катализаторами или компонентами каталитических систем в реакциях превращения органических соединений в жидкой фазе [1-3]. При изучении механизма каталитических реакций контроль состава исходных ПОМ и ИЖ, а также наблюдение за их превращениями в условиях реакции осуществляются с использованием методов физикохимического анализа. В данной работе представлены результаты наших исследований жидкофазных каталитических систем, содержащих ПОМ и ИЖ, с использованием методов элементного анализа, в особенности CHNSанализа, в сочетании со спектрами гетерополианионов ЯМР ${ }^{31} \mathrm{P}$ и УФ-ВИД.

В данной работе применен CHNS-анализ для определения количества и состава органических катионов, разбавленных другими компонентами катализаторов или растворителем. В одном случае анализировали катионы соли с крупными по массе вольфрамофосфат-анионами 
в катализаторах или их предшественниках, а в другом - малые количества и состав органических катионов определяли в реакционном растворе. В этих двух примерах мы имели дело с меньшими количествами определяемых элементов, чем это обычно имеет место при идентификации индивидуальных органических соединений. В процессе решения поставленных задач проведена также оценка возможностей определения с помощью CHNS-анализа малых количеств ИЖ в растворе летучих соединений.

\section{Экспериментальная часть}

\section{Реактивы и материаль}

В работе использовали тетрабутиламмоний бромид $\left(\mathrm{Bu}_{4} \mathrm{NBr}\right.$, “Реахим"), 1-этил-3метилимидазолий бромид ([EMIm]Br, “Aldrich”), 1-бутил-3-метилимдазолий бромид ([BMImBr], “Aldrich”), тетрабутиламмоний гидросульфат $\left(\mathrm{Bu}_{4} \mathrm{NHSO}_{4}\right.$, “PS”), гетерополикислоту $\left(\mathrm{H}_{3} \mathrm{PMo}_{12} \mathrm{O}_{40} \cdot 9 \mathrm{H}_{2} \mathrm{O}\right.$, “Реахим”), очищенную с помощью эфирной экстракции, ацетонитрил ( $\mathrm{CH}_{3} \mathrm{CN}$, “Aldrich”), 1,3-бутадиен (“Aldrich”), циклогексан (ч.д.а., “Реахим”), перекись водорода 34\%-ный водный раствор (“Реахим”). Применяли растертые образцы носителей: силикагель марки КСК $\left(\mathrm{S}_{\text {пов }}=263 \mathrm{~m}^{2} \Gamma^{-1}, \mathrm{~V}_{\text {пор }}=0.84 \mathrm{~cm}^{3} \Gamma^{-1}\right.$, прокаленный при $500{ }^{\circ} \mathrm{C}$, содержащий примеси $0.81 \% \mathrm{Na}, 0.54 \% \mathrm{Ca}, 0.35 \% \mathrm{Al}, 0.10 \% \mathrm{Mg}, 0.06 \% \mathrm{Fe}, 0.06 \% \mathrm{~K}, 0.04 \% \mathrm{Cl}, 0.01 \% \mathrm{~S}$ ) и оксид кремния MS-3050 (PQ Corp. $\mathrm{S}_{\text {пов }}=537 \mathrm{M}^{2} \Gamma^{-1}, \mathrm{~V}_{\text {пор }}=3.0 \mathrm{~cm}^{3} \Gamma^{-1}$, содержащий $0.01 \% \mathrm{Na}$ ).

\section{Приготовление ПОМ}

Рассмотренные в работе соли вольфрамофосфат-аниона с органическими катионами готовили исходя из раствора $\mathrm{Na}$-соли $\mathrm{PW}_{11} \mathrm{O}_{39}{ }^{7-}$ в воде при $\mathrm{pH} 4$ добавлением избытка $\mathrm{Bu}_{4} \mathrm{NBr}$ или [BMIm] $\mathrm{Br}$, как описано в нашей работе [4]. $\mathrm{Bu}_{4} \mathrm{~N}^{+}$-соли пероксокомплексов вольфрамофосфат-анионов синтезированы по известным методикам $[5,6]$. Соли М-замещенных вольфрамофостат-анионов $\left[\mathrm{PW}_{11} \mathrm{O}_{39} \mathrm{Cu}\left(\mathrm{H}_{2} \mathrm{O}\right)\right]^{5-}$ и $\left[\mathrm{PW}_{11} \mathrm{O}_{39} \mathrm{Fe}(\mathrm{OH})\right]^{5-}$ с имидазолиевыми катионами получали осаждением из водных растворов при добавлении [EMIm] Br или [BMIm] Br [7]. Состав катионов полученных соединений по данным CHNS-анализа приведен в табл. 1 и 2. Анализ ПОМ на содержание P осуществляли спектрофотометрически по образованию P-Mo-V комплекса, W и Na определяли методом атомно-эмиссионной спектрометрии с индуктивно-связанной плазмой (ИСП-АЭС). Мо в нанесенных на силикагель образцах выявляли рентгеновским флуоресцентным методом.

Таблица 1. Результаты C, $\mathrm{H}, \mathrm{N}$ анализа $\mathrm{Cu}^{2+}$ и $\mathrm{Fe}^{3+}$ замещенных вольфрамофосфатов Table 1. The $\mathrm{C}, \mathrm{H}, \mathrm{N}$ analysis of $\mathrm{Cu}^{2+}$ and $\mathrm{Fe}^{3+}$ substituted tungstophosphates

\begin{tabular}{|c|l|cccc|}
\hline \multicolumn{1}{|c|}{ № } & \multicolumn{1}{|c|}{ Состав } & & $\mathrm{C}$, & $\mathrm{H}$, & $\mathrm{N}, \%$ \\
\hline \multirow{2}{*}{1} & {$\left[\left(\mathrm{C}_{2} \mathrm{H}_{5}\right) \mathrm{CH}_{3} \mathrm{C}_{3} \mathrm{H}_{3} \mathrm{~N}_{2}\right]_{5}\left[\mathrm{PW}_{11} \mathrm{O}_{39} \mathrm{Cu}\left(\mathrm{H}_{2} \mathrm{O}\right)\right]$} & рассч. & 10.86 & 1.73 & 4.22 \\
& м.в. 3315 & найд. & 10.7 & 1.80 & 4.18 \\
\hline \multirow{2}{*}{2} & {$\left[\left(\mathrm{C}_{4} \mathrm{H}_{9}\right) \mathrm{CH}_{3} \mathrm{C}_{3} \mathrm{H}_{3} \mathrm{~N}_{2}\right]_{5}\left[\mathrm{PW}_{11} \mathrm{O}_{39} \mathrm{Cu}\left(\mathrm{H}_{2} \mathrm{O}\right)\right]$} & рассч. & 13.9 & 2.24 & 4.05 \\
& м.в. 3455 & найд. & 13.8 & 2.23 & 4.01 \\
\hline \multirow{2}{*}{3} & {$\left[\left(\mathrm{C}_{4} \mathrm{H}_{9}\right) \mathrm{CH}_{3} \mathrm{C}_{3} \mathrm{H}_{3} \mathrm{~N}_{2}\right]_{5}\left[\mathrm{PW}_{11} \mathrm{O}_{39} \mathrm{Fe}(\mathrm{OH})\right]$} & рассч. & 13.9 & 2.22 & 4.06 \\
& м.в. 3446 & найд. & 13.6 & 2.25 & 4.01 \\
\hline
\end{tabular}


Таблица 2. Элементный состав синтезированных вольфрамофосфатов, предшественников катализаторов эпоксидирования 1,3-бутадиена перекисью водорода

Table 2. The elemental composition of the synthesized tungstophosphates, the precursors of catalysts for epoxidation of 1,3-butadiene with hydrogen peroxide

\begin{tabular}{|c|c|c|c|c|c|c|c|}
\hline № & \multicolumn{7}{|c|}{ Соединение } \\
\hline \multirow{4}{*}{1} & \multicolumn{7}{|c|}{$\left[\left(\mathrm{C}_{4} \mathrm{H}_{9}\right)_{4} \mathrm{~N}\right]_{5} \mathrm{Na}_{0.55} \mathrm{H}_{1.45}\left[\mathrm{PW}_{11} \mathrm{O}_{39}\right]\left(\mathrm{Bu}_{4} \mathrm{~N}-\mathrm{PW}_{11}\right)$ м.в. 3901} \\
\hline & & $\mathrm{C}$ & $\mathrm{H}$ & $\mathrm{N}$ & $\mathrm{P}$ & $\mathrm{W}$, & $\mathrm{Na}, \%$ \\
\hline & рассч. & 24.6 & 4.65 & 1.79 & 0.795 & 51.8 & 0.32 \\
\hline & найд. & 24.2 & 4.55 & 1.70 & 0.674 & 50.8 & 0.32 \\
\hline \multirow{4}{*}{2} & \multicolumn{7}{|c|}{$\left.\left[\left(\mathrm{C}_{2} \mathrm{H}_{5}\right) \mathrm{CH}_{3}\right) \mathrm{C}_{3} \mathrm{H}_{3} \mathrm{~N}_{2}\right]_{5} \mathrm{Na}_{1.35} \mathrm{H}_{0.65}\left[\mathrm{PW}_{11} \mathrm{O}_{39}\right]\left(\mathrm{EMIm}-\mathrm{PW}{ }_{11}\right)$ м.в. 3264} \\
\hline & & $\mathrm{C}$ & $\mathrm{H}$ & $\mathrm{N}$ & $\mathrm{P}$ & $\mathrm{W}$ & $\mathrm{Na}, \%$ \\
\hline & рассч. & 11.03 & 1.71 & 4.29 & 0.950 & 62.1 & 0.95 \\
\hline & найд. & 11.1 & 2.10 & 4.30 & 0.891 & 57.7 & 0.94 \\
\hline \multirow{4}{*}{3} & \multicolumn{7}{|c|}{$\left.\left[\left(\mathrm{C}_{4} \mathrm{H}_{9}\right)_{4} \mathrm{~N}\right]_{3}\left\{\mathrm{PO}_{4}\left[\mathrm{WO}\left(\mathrm{O}_{2}\right)_{2}\right]_{4}\right\}\left(\mathrm{Bu}_{4} \mathrm{~N}-\mathrm{PW}\right)_{4}\right)$ м.в. 1876} \\
\hline & & $\mathrm{C}$ & $\mathrm{H}$ & $\mathrm{N}$ & $\mathrm{P}$, & $\mathrm{W}, \%$ & \\
\hline & рассч. & 30.7 & 5.75 & 2.24 & 1.65 & 39.2 & \\
\hline & найд. & 31.4 & 5.90 & 2.24 & 1.64 & 38.7 & \\
\hline \multirow{4}{*}{4} & \multicolumn{7}{|c|}{$\left[\left(\mathrm{C}_{4} \mathrm{H}_{9}\right)_{4} \mathrm{~N}\right]_{2}\left\{\mathrm{HPO}_{4}\left[\mathrm{WO}\left(\mathrm{O}_{2}\right)_{2}\right]_{2}\right\}\left(\mathrm{Bu}_{4} \mathrm{~N}-\mathrm{PW}_{2}\right)$ м.в. 1108} \\
\hline & & $\mathrm{C}$ & $\mathrm{H}$ & $\mathrm{N}$ & $\mathrm{P}$, & $\mathrm{W}, \%$ & \\
\hline & рассч. & 34.7 & 6.59 & 2.53 & 2.80 & 33.2 & \\
\hline & найд. & 34.4 & 6.50 & 2.60 & 2.55 & 33.2 & \\
\hline
\end{tabular}

\section{Методика CHNS-анализа}

Анализ осуществляли на CHNS-анализаторе "Euro EA 3000” (“Eurovector”, Италия). Содержание элементов определяли исходя из площади хроматографических пиков $\mathrm{N}_{2}, \mathrm{CO}_{2}, \mathrm{H}_{2} \mathrm{O}$ и $\mathrm{SO}_{2}$ по калибровочной прямой, построенной по стандартным соединениям: цистеина, атропинсульфата, сульфаниламида и L-цистина. Обработку результатов вели по оригинальной программе Callidus.

Для определения малых количеств ИЖ ацетонитрильный раствор (1.5 мл), содержащий 20 - 40 мкмолей ИЖ и другие компоненты каталитической системы $\left(\mathrm{H}_{3} \mathrm{PMo}_{12} \mathrm{O}_{40}\right.$, циклогексан, циклогексанол и воду), добавляли к навеске оксида кремния (в районе 0.25 г), прогретого при $170{ }^{\circ} \mathrm{C}$. Растворитель испаряли на воздухе при тщательном перемешивании, затем образец прогревали в течение 1 ч при $170{ }^{\circ} \mathrm{C}$ для удаления оставшихся летучих соединений. Приготовленные образцы держали в эксикаторе.

Навески анализируемых образцов изменяли в зависимости от предполагаемого содержания элементов в пределах 4-15 мг.

\section{Результаты и их обсуждение}

\section{1. Соотнесение состава и каталитической активности вольфрамофосфатов} в реакциях перекисного окисления

Гетерополивольфраматы являются катализаторами широкого круга реакций, основанных на активации $\mathrm{H}_{2} \mathrm{O}_{2}$. В данной части работы наше внимание было сосредоточено на выявле- 
нии связи каталитических свойств вольфрамофосфатов с особенностями их состава и строения. Исследуемые ПОМ представляли собой соли тетрабутиламмоний или имидазолий катионов и вольфрамофосфат-анионов, растворимые в реакционной среде. Структурный тип и методы получения этих ПОМ известны, а сочетание элементного анализа с ЯМР и УФ-ВИДспектроскопией является традиционным способом исследования их состава и строения в растворах. Мы применили эти методы для определения исходного состояния ПОМ, а также для контроля изменений их состава и строения в условиях реакции $[4,7]$.

Возможности $\mathrm{CHN}$-анализа полиоксометаллатов демонстрирует табл. 1. Процентное содержание $\mathrm{C}, \mathrm{H}$ и $\mathrm{N}$ в полученных М-замещенных вольфрамофосфатах отражает даже небольшие изменения состава катиона в соединениях № 1 и 2. Содержание $\mathrm{C}, \mathrm{H}$ и $\mathrm{N}$ в образцах № 1-3 рассчитывали в предположении сохранения исходного фрагмента $\mathrm{PW}_{11} \mathrm{O}_{39}{ }^{7}$, находящегося в составе более сложного металлсодержащего аниона. Результаты анализа согласуются с таким предположением и подтверждают, что структура исходного вольфрамофосфата не разрушается в процессе синтеза металлсодержащих анионов. Это заключение важно для катализа, поскольку дает нам основание относить разницу в каталитических свойствах этих ПОМ к влиянию металла М. Известно [8], что в присутствии $\mathrm{H}_{2} \mathrm{O}_{2}$ анионы $\left[\mathrm{PW}_{11} \mathrm{O}_{39} \mathrm{Fe}(\mathrm{OH})\right]^{5-}$ слабо катализируют разложение перекиси водорода и образуют окрашенный комплекс (УФ-ВИД: $\lambda=380-550$ нм), а в реакции с циклогексеном были получены циклогексенол и циклогексенон с примесью эпоксида. Наоборот, анионы $\left[\mathrm{PW}_{11} \mathrm{O}_{39} \mathrm{Cu}\left(\mathrm{H}_{2} \mathrm{O}\right)\right]^{5-}$ лишь интенсивно разлагают $\mathrm{H}_{2} \mathrm{O}_{2}$ без окисления циклогексена.

Для изучения каталитических свойств ПОМ в эпоксидировании 1,3-бутадиена перекисью водорода нами синтезированы соли $\left[\mathrm{PW}_{11} \mathrm{O}_{39}\right]^{7-}$ аниона (табл. 2, № 1 и 2) и известные в литературе $[5,6]$ пероксокомплексы (табл. 2, № 3 и 4). Выполненный элементный анализ соединений соответствует приведенным формулам ПОМ. Лишь в единичных случаях получено заниженное содержание Р (№ 1 и 4), что, по-видимому, связано с точностью метода определения Р.

Методом ИСП-АЭС было обнаружено, что образцы 1 и 2 содержат Na. Принятые в расчет количества $\mathrm{Na}$ соответствуют брутто формулам соединений, которые приведены в табл. 2. Структура анионов и вхождение ионов Nа в состав полученных ПОМ подтверждены с помощью ЯМР ${ }^{31} \mathrm{P}$. Спектр $\mathrm{Bu}_{4} \mathrm{~N}-\mathrm{PW}_{11}$, растворенного в ацетонитриле, имеет два широких сигнала с $\delta=-12.3$ и -12.8 м.д., которые переходят в один узкий пик (-12.5 м.д.) при добавлении воды $\left(\mathrm{CH}_{3} \mathrm{CN} / \mathrm{H}_{2} \mathrm{O}=10 / 1\right.$, об.) [4]. Полученные сигналы относятся к полианионам $\left[\mathrm{HPW}_{11} \mathrm{O}_{39}\right]^{6-}$ и $\left[\mathrm{H}_{2} \mathrm{PW}_{11} \mathrm{O}_{39}\right]^{5-}$ и сливаются в один узкий сигнал в присутствии воды вследствие быстрого протонного обмена [9]. Соотношение интенсивностей этих двух сигналов, равное $\sim$ 1.4:1, соответствует среднему числу ионов натрия в соли $\mathrm{Bu}_{4} \mathrm{~N}-\mathrm{PW}_{11}$, найденному по химанализу. Следует заметить, что кристаллизация смешанных кислых солей, таких как $\left.\mathrm{Na}_{2}\left[\mathrm{~N}\left(\mathrm{CH}_{3}\right)_{4}\right]\right]_{4} \mathrm{H}\left[\mathrm{PW}_{11} \mathrm{O}_{39}\right] \cdot 7 \mathrm{H}_{2} \mathrm{O}$ [10], характерна для объемных органических катионов вследствие стерических затруднений. Таким образом, данные химанализа в сочетании со спектрами ЯМР ${ }^{31} \mathrm{P}$ дают состав ПОМ предшественников катализаторов.

Далее, при снятии спектров ЯМР ${ }^{31} \mathrm{P}$ в процессе реакции эпоксидирования 1,3-бутадиена Р.И. Максимовская [4] продемонстрировала связь между составом синтезированных предшественников и образующихся в реакционных условиях ПОМ, которые изображены на

$$
-225-
$$


1

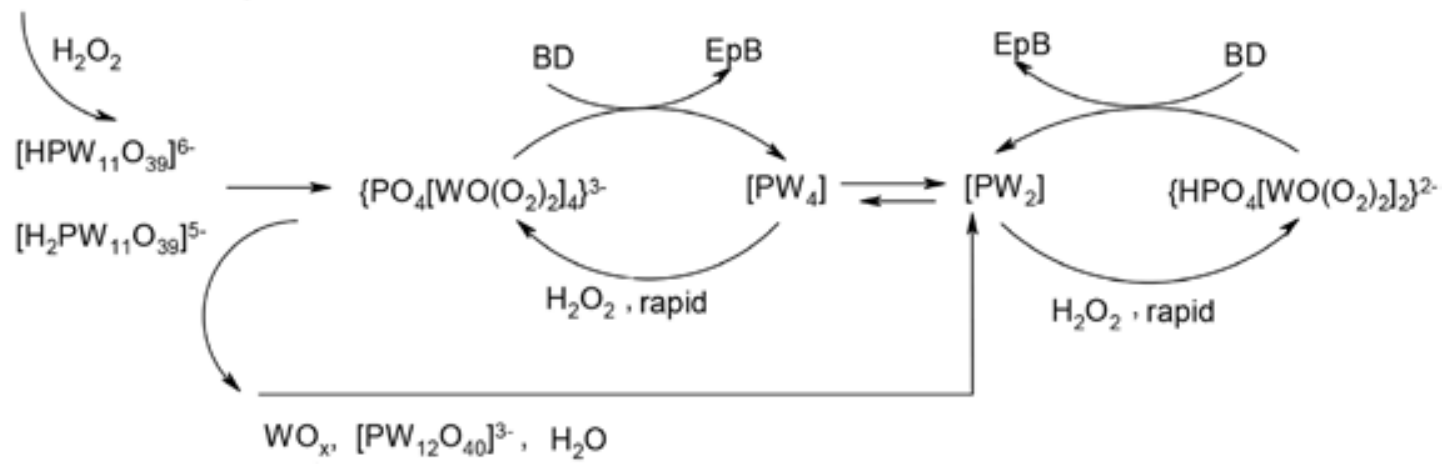

Рис. 1. Превращения анионов предшественника в ацетонитрильном растворе, содержащем водную $\mathrm{H}_{2} \mathrm{O}_{2}$ (1), во время начального (2) и близкого к стационарному (3) периодам реакции окисления 1,3-бутадиена (BD) в 3,4-эпокси-1-бутен (ЕрВ)

Fig. 1. Transformations of the precursor anions in acetonitrile solution containing aqueous $\mathrm{H}_{2} \mathrm{O}_{2}$ (1), during the initial (2) and close to stationary (3) periods of 1,3-butadiene oxidation into 3,4-epoxy-1-butene

pис. 1. При использовании в качестве предшественника EMIm-PW 11 наблюдалось повышенное по сравнению с TBA-PW 11 образование активных пероксоанионов $\left\{\mathrm{PO}_{4}\left[\mathrm{WO}\left(\mathrm{O}_{2}\right)_{2}\right]_{4}\right\}^{3-}$ и $\left\{\mathrm{HPO}_{4}\left[\mathrm{WO}\left(\mathrm{O}_{2}\right)_{2}\right]_{2}\right\}^{2-}$, и при этом увеличивалась активность каталитической системы в реакции эпоксидирования бутадиена.

\section{2. определение с помощью CHNS-анализа малых конщентраций ИЖ}

в реакционных растворах для окисления ијилогексана смесью $\mathrm{O}_{2}-\mathrm{H}_{2}$

В каталитических экспериментах было обнаружено, что скорость окисления циклогексана смесью газов $\mathrm{O}_{2}-\mathrm{H}_{2}$ до циклогексанола и селективность реакции увеличиваются, когда вместе с двухкомпонентным катализатором $\mathrm{Pt} / \mathrm{C}+\mathrm{H}_{3} \mathrm{PMo}_{12} \mathrm{O}_{40}$ в реакционную среду вводили ИЖ $\mathrm{Bu}_{4} \mathrm{NBr}$, [BMIm] Br, $\mathrm{Bu}_{4} \mathrm{NHSO}_{4}$ [11]. Для выяснения природы наблюдаемого эффекта необходимо было изучить распределение ИЖ между реакционным раствором, содержащим $\mathrm{H}_{3} \mathrm{PMo}_{12} \mathrm{O}_{40}$ и органические компоненты в ацетонитриле, и поверхностью твердого катализатора $\mathrm{Pt} / \mathrm{C}$. Количества ИЖ определяли в исходных растворах до и после контакта c $\mathrm{Pt} / \mathrm{C}$, a затем после проведения каталитической реакции. С этой целью известные порции растворов испаряли на силикагеле марки КСК и после прогрева образца для удаления летучих компонентов проводили анализ. Выбор силикагеля, процедуры нанесения ИЖ, а также условий термической обработки образцов перед анализом подробно описаны в нашей предыдущей работе [12]. Результаты анализа образцов, содержащих $3.5-4.5$ вес. \% ИЖ, приведены в табл. 3 .

Найденные содержания $\mathrm{N}, \mathrm{C}$, а ткже Мо в образцах, приготовленных из исходных растворов перед контактом с Pt/C, совпадали с вычисленными исходя из взятого количества реагентов (табл. 3, № 1 и 4). Судя по найденным и вычисленным значениям N и C, после контакта раствора с Pt/C изменения в концентрации растворенных ИЖ (табл. 3, № 2, 5 и 7) были соизмеримы с экспериментальной ошибкой, что свидетельствует об очень низкой адсорбции катионов ИЖ. Интересно, что столь низкая степень адсорбции ИЖ вызывала тем не менее влияние на 
Таблица 3. Анализ твердых образцов, приготовленных путем нанесения на силикагель реакционных растворов $^{\text {a }}$

Table 3. Analysis of solid samples prepared by applying reaction solutions to silica gel ${ }^{\mathrm{a}}$

\begin{tabular}{|c|c|c|c|c|c|c|c|c|c|}
\hline \multirow{2}{*}{ № } & \multirow{2}{*}{ ИЖ } & \multirow{2}{*}{$\begin{array}{c}\text { ИЖ в } \\
\text { исходном } \\
\text { растворе, г }\end{array}$} & \multirow{2}{*}{ Процедура } & \multicolumn{2}{|c|}{$\mathrm{N}$, вес. $\%$} & \multicolumn{2}{|c|}{ С, вес. $\%$} & \multicolumn{2}{|c|}{ Мо, вес. \% } \\
\hline & & & & найд. & выч. & найд. & выч. & найд. & выч. \\
\hline 1 & $\mathrm{Bu}_{4} \mathrm{NBr}$ & 0.0180 & $\mathrm{~A}$ & 0.197 & 0.193 & 2.69 & 2.65 & 2.85 & 2.86 \\
\hline 2 & -“" & 0.0180 & Б & 0.188 & 0.193 & 2.55 & 2.65 & 2.20 & 2.86 \\
\hline 3 & -“ - & 0.0179 & B & 0.192 & 0.192 & 2.65 & 2.64 & 2.72 & 2.86 \\
\hline 4 & $\mathrm{Bu}_{4} \mathrm{NHSO}_{4}$ & 0.0194 & A & 0.201 & 0.198 & 2.71 & 2.71 & 2.96 & 2.85 \\
\hline 5 & -" - & 0.00975 & Б & 0.106 & 0.102 & 1.40 & 1.39 & 2.52 & 2.92 \\
\hline 6 & -“" & 0.0189 & B & 0.176 & 0.183 & 2.83 & 2.52 & 4.13 & 5.45 \\
\hline 7 & BMImBr & 0.0140 & Б & 0.430 & 0.442 & 1.51 & 1.55 & 2.36 & 2.89 \\
\hline 8 & -“" & 0.0129 & B & 0.375 & 0.411 & 1.38 & 1.41 & 2.48 & 2.90 \\
\hline
\end{tabular}

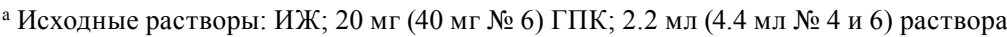

$\mathrm{CH}_{3} \mathrm{CN} /$ циклогексан = 10/1 (мл/мл); объем раствора, взятого для анализа, 1.5 мл (3.0 мл № 4 и 6) на 0.2500 г силикагеля KCK;

${ }^{\sigma} \mathrm{A}$ - перед добавлением катализатора; Б - после контакта с 20 мг Pt/C в течение 1 ч на воздухе; В - после проведения каталитической реакции.

катализ. Мы пришли к заключению, что это влияние $\mathrm{Br}$-содержащих ИЖ связано с модификацией поверхности нанесенной $\mathrm{Pt}$, для чего достаточно было малых количеств $\mathrm{Br}$ анионов на поверхности $\mathrm{Pt} / \mathrm{C}$ [13]. Изменения концентрации ИЖ в растворе не наблюдались и после проведения каталитической реакции (табл. 3, № 3, 6, 8). Атомные отношения $\mathrm{C} / \mathrm{N}$ в ИЖ оставались неизменными, что подтверждает стабильность органического катиона в условиях реакции. Заметим, что результаты анализа водорода не использовали для определения количеств ИЖ, так как в процессе сжигания проб происходило выделение небольших количеств структурной воды из силикагеля и в результате разложения $\mathrm{H}_{3} \mathrm{PMo}_{12} \mathrm{O}_{40}$ [12]. Небромидная ИЖ $\mathrm{Bu}_{4} \mathrm{NHSO}_{4}$ также оказывала промотирующий эффект на каталитическую систему, но действовала иным путем. Кислотный характер аниона $\mathrm{HSO}_{4}{ }^{-}$, по-видимому, способствовал образованию перекиси водорода как активного окислителя в растворе. В то же время, как показал ИСП-АЭС анализ раствора, присутствие $\mathrm{Bu}_{4} \mathrm{NHSO}_{4}$ не приводило к растворению платины. В результате применение $\mathrm{Bu}_{4} \mathrm{NHSO}_{4}$ позволило достичь наилучших для данной системы показателей производительности и селективности.

Анализ на Мо показал, что от 5 до $\sim 20 \%$ от исходного количества $\mathrm{H}_{3} \mathrm{PMo}_{12} \mathrm{O}_{40}$ адсорбировались на поверхности твердого катализатора $\mathrm{Pt} / \mathrm{C}$. При этом часть оставшейся в растворе гетерополикислоты находилась в восстановленном состоянии (УФ-ВИД: $\lambda_{\max }=700$ и 875 нм). По спектрам ЯМР ${ }^{31} \mathrm{P}$ удалось наблюдать сигналы, относящиеся [14] к окисленной $\mathrm{PMo}_{12} \mathrm{O}_{40}{ }^{3-}$ (-4 м.д.) и к восстановленной на 2 электрона $\mathrm{H}_{\mathrm{x}} \mathrm{PMo}_{12} \mathrm{O}_{40}{ }^{\mathrm{x}-5}$ (-6.8 м.д.). С учетом распределения $\mathrm{H}_{3} \mathrm{PMo}_{12} \mathrm{O}_{40}$ и ИЖ между раствором и поверхностью твердого $\mathrm{Pt} / \mathrm{C}$ компонента предложена схема (рис. 2), описывающая механизм реакции окисления циклогексана в циклогексанол с использованием смеси $\mathrm{O}_{2}-\mathrm{H}_{2}$ [15]. Активация молекулы кислорода идет на поверхности нанесенной платины. ИЖ способствуют стабилизации перекисного кислорода и образованию $\mathrm{H}_{2} \mathrm{O}_{2}$. Окисление субстрата перекисью водорода происходит в растворе на ионах молибдена гетерополикислоты. 


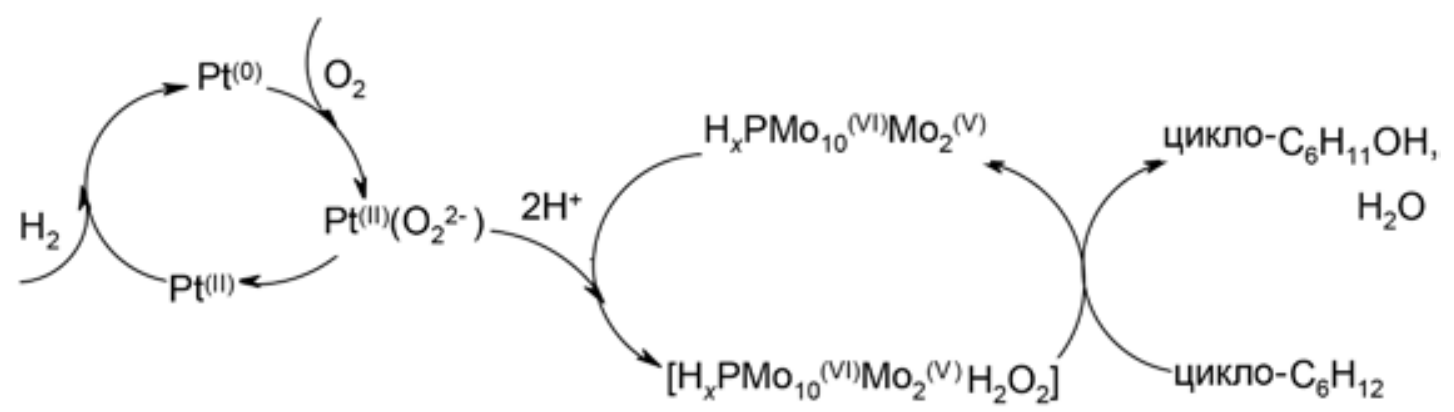

Рис. 2. Окисление циклогексана смесью $\mathrm{O}_{2}-\mathrm{H}_{2}$ в каталитической системе $\mathrm{Pt} / \mathrm{C}-\mathrm{H}_{3} \mathrm{PMo}_{12} \mathrm{O}_{40}-\mathrm{Bu}_{4} \mathrm{NHSO}_{4}-$ $\mathrm{CH}_{3} \mathrm{CN}$

Fig. 2. Oxidation of cyclohexane with a mixture of $\mathrm{O}_{2}-\mathrm{H}_{2}$ in the catalytic system $\mathrm{Pt} / \mathrm{C}-\mathrm{H}_{3} \mathrm{PMo}_{12} \mathrm{O}_{40}-\mathrm{Bu}_{4} \mathrm{NHSO}_{4}-$ $\mathrm{CH}_{3} \mathrm{CN}$

\section{3. Оиенка возможностей метода CHNS-анализа для определения ИЖ в растворах летучих соединений}

Для характеризации индивидуальных ИЖ наряду с ЯМР и ИК-спектроскопией обычно используют метод CHNS-анализа [16], тогда как методы ВЭЖХ [17] и КЭФ [18] подходят для определения концентрации ИЖ в растворе. Оригинальный способ проведения CHNS-анализа каталитических растворов с низким содержанием ИЖ позволил нам получать корректную информацию о концентрации ИЖ и сохранении состава органических катионов в процессе каталитических испытаний по результатам C и $\mathrm{N}$ анализа, описанного в [12]. Объективность данного метода проверена нами в предварительных экспериментах. Мы приготовили несколько образцов путем пропитки навески образцов пористого оксида кремния КСК и MS-3050 растворами ИЖ. Результаты анализа $\mathrm{Bu}_{4} \mathrm{NBr}$ и [BMIm] $\mathrm{Br}$ приведены в табл. 4. Для всех образцов получена хорошая воспроизводимость в параллельных пробах. Найденное содержание $\mathrm{C}$ и $\mathrm{N}$ соответствовало вычисленному по навеске введенному количеству ИЖ. Относительные откло-

Таблица 4. Анализ твердых образцов, приготовленных путем нанесения на оксид кремния КСК или MS3050 ацетонитрильных растворов ИЖ ${ }^{\text {a }}$

Table 4. Analysis of solid samples prepared by applying acetonitrile solutions of IL to silica KCK or MS-3050 a

\begin{tabular}{|c|c|c|c|c|c|c|c|}
\hline \multirow{2}{*}{ № } & \multirow{2}{*}{ Состав } & \multicolumn{2}{|c|}{ C, вес. $\%$} & \multicolumn{2}{|c|}{$\mathrm{N}$, вес. $\%$} & \multicolumn{2}{|c|}{ H, вес. $\%$} \\
\hline & & найд. & выч. & найд. & выч. & найд. & выч. \\
\hline 1 & $\left(\mathrm{C}_{4} \mathrm{H}_{9}\right)_{4} \mathrm{NBr}, \mathrm{KCK}$ & $3.629 \pm 0.027$ & 669 & $0.266 \pm 0.002$ & 0.267 & $0.93 \pm 0.01$ & 0.722 \\
\hline 2 & $\left(\mathrm{C}_{4} \mathrm{H}_{9}\right)_{4} \mathrm{NBr}, \mathrm{MS}-3050$ & $3.949 \pm 0.002$ & 3.880 & $0.289 \pm 0.003$ & 0.283 & $1.500 \pm 0.013$ & 0.727 \\
\hline 3 & $\begin{array}{l}\left(\mathrm{C}_{4} \mathrm{H}_{9}\right)\left(\mathrm{CH}_{3}\right) \mathrm{N}_{2} \mathrm{C}_{3} \mathrm{H}_{3} \mathrm{Br}, \\
\mathrm{KCK}\end{array}$ & $2.398 \pm 0.003$ & 2.396 & $0.688 \pm 0.002$ & 0.699 & $0.590 \pm 0.003$ & 0.397 \\
\hline 4 & $\begin{array}{l}\left(\mathrm{C}_{4} \mathrm{H}_{9}\right)\left(\mathrm{CH}_{3}\right) \mathrm{N}_{2} \mathrm{C}_{3} \mathrm{H}_{3} \mathrm{Br} \\
\mathrm{MS}-3050\end{array}$ & $8.43 \pm 0.01$ & 8.34 & $2.43 \pm 0.01$ & 2.43 & $2.20 \pm 0.07$ & 1.30 \\
\hline $5^{\sigma}$ & $\left(\mathrm{C}_{7} \mathrm{H}_{7}\right)\left(\mathrm{C}_{4} \mathrm{H}_{9}\right) \mathrm{N}_{2} \mathrm{C}_{3} \mathrm{H}_{3} \mathrm{Br}$ & 56.31 & 56.96 & 9.61 & 9.49 & 6.38 & 6.49 \\
\hline
\end{tabular}

а Объем раствора 1.5 мл, навески ИЖ около 0.015 г, навески силикагеля около 0.25 г; навески образцов для анализа $5-7$ мг;

б чистый 1-бензил-3-бутилимидазолий бромид, по данным работы [16]. 
Таблица 5. Результаты C, N, S анализа ИЖ $\left[\left(\mathrm{C}_{4} \mathrm{H}_{9}\right)_{4} \mathrm{~N}\right] \mathrm{HSO}_{4}$, нанесенной на оксид кремния КСК (№ 1 и 2) или MS-3050 (№ 3 и 4) ${ }^{\text {a }}$

Table 5. The results of $\mathrm{C}, \mathrm{N}, \mathrm{S}$ analysis of $\mathrm{IL}\left[\left(\mathrm{C}_{4} \mathrm{H}_{9}\right)_{4} \mathrm{~N}\right] \mathrm{HSO}_{4}$ applied on silica KSK (No. 1 and 2) or MS-3050 $(\text { No. } 3 \text { and } 4)^{\text {a }}$

\begin{tabular}{|c|cc|c|cc|c|ccc|}
\hline \multirow{2}{*}{ № } & \multicolumn{3}{|c|}{ С, вес. \% } & \multicolumn{3}{c|}{ N, вес. \% } & \multicolumn{3}{c|}{ S, вес. \% } \\
\cline { 2 - 11 } & \multicolumn{2}{|c|}{ найд. } & выч. & \multicolumn{2}{c|}{ найд. } & выч. & \multicolumn{2}{c|}{ найд. } & выч. \\
\hline 1 & $1.73 ;$ & 1.74 & 1.82 & $0.132 ;$ & 0.138 & 0.133 & $0.277 ;$ & 0.260 & 0.304 \\
\hline 2 & $2.90 ;$ & 2.90 & 3.01 & $0.215 ;$ & 0.219 & 0.220 & $0.437 ;$ & 0.387 & 0.502 \\
\hline 3 & $3.23 ;$ & 3.19 & 3.16 & $0.248 ;$ & 0.246 & 0.231 & $0.430 ;$ & 0.408 & 0.527 \\
\hline 4 & $9.99 ;$ & 9.91 & 10.1 & $0.750 ;$ & 0.748 & 0.735 & $1.53 ;$ & 1.45 & 1.68 \\
\hline
\end{tabular}

а Объем ацетонитрильного раствора 1.5 мл, навески ИЖ варьировали в пределах $0.007-0.04$ г, навески силикагеля около 0.20 г; по двум параллельным измерениям, навески образцов 5 - 15 мг.

нения от определяемых величин не превышали $\pm 2 \%$, т.е. не отличались от таковых для чистой ИЖ близкого состава, также приведенной в табл. 4. Завышенные содержания Н в нанесенных образцах, как уже отмечалось, связаны с присутствием структурной воды в носителях.

$\mathrm{Bu}_{4} \mathrm{NHSO}_{4}$ также наносили на два разных типа оксида кремния и при изменении количества нанесенной ИЖ. Результаты анализа (табл. 5) показали удовлетворительное для данного уровня содержания определяемых элементов совпадение полученных результатов определения $\mathrm{C}, \mathrm{N}$ и $\mathrm{S}$ с рассчитанными для этих образцов.

\section{Заключение}

Показаны возможности CHNS-анализа при определении состава ПОМ и концентрации ИЖ в каталитических системах окисления углеводородов в жидкой фазе.

В рамках уже известного структурного типа вольфрамофосфат-анионов с катионами TBA или имидазолия результаты элементного анализа отражают особенности состава приготовленных соединений. Далее, с помощью спектроскопии ЯМР ${ }^{31} \mathrm{P}$ в ацетонитрильном растворе получены сведения о протонировании анионов $\mathrm{PW}_{11} \mathrm{O}_{39}{ }^{7-}$ в исходном состоянии, о их превращении в каталитически активные пероксоанионы и о лучшей стабилизации последних катионами имидазолия.

Предложенный способ $\mathrm{C}$ и $\mathrm{N}$ анализа растворов ИЖ позволил определить распределение

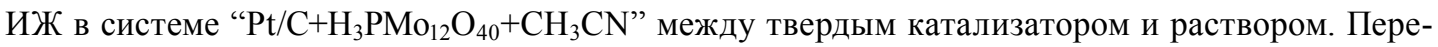
кисный кислород образуется на поверхности Рt и при участии ИЖ. В то же время по спектрам УФ-ВИД и ЯМР ${ }^{31} \mathrm{P}$ активная в перекисном окислении циклогексана частично восстановленная гетерополикислота определена в реакционном растворе.

\section{Список литературы / References}

1. Katsoulis D.E., Pope M.T. Reactions of heteropolyanions in non-polar solvents. Part 3. Activation of dioxygen by manganese(II) centres in polytungstates. Oxidation of hindered phenols. J. Chem. Soc. Dalton Trans. 1989, P. 1483-1489.

2. Wang S.-S., Yang G.-Y. Recent advances in polyoxometelate. Chem. Rev. 2015. Vol. 115(11), P. 4893-4962. 
3. Xue S., Chen G., Long Z., Zhou Y., Wang J. Efficient and recyclabe multi-cationic polyoxometalate-based hybrid catalyst for heterogeneous cyclohexane oxidation with $\mathrm{H}_{2} \mathrm{O}_{2} \cdot R S C A d v$. 2015. Vol. 5, P. 19306-19314.

4. Kuznetsova L.I., Kuznetsova N.I., Maksimovsraya R.I., Aleshina G.I., Koscheeva O.S., Utkin V.A. Epoxidation of butadiene with hydrogen peroxide catalyzed by the salts of phosphotungstste anions: relation betveen catalytic activity and composition of intermediate peroxo complexes. Catal. Lett. 2011. Vol. 141, P. 1442-1450.

5. Aubry C., Chottard G., Platzer N., Brégeault J.-M., Thouvenot R., Chauveau F., Huet C., Ledon H. Reinvestigation of epoxidation using tungsten-based precursors and hydrogen peroxide in a biphase medium. Inorg. Chem. 1991. Vol. 30(23), P. 4409-4415.

6. Salles L., Aubry C., Thouvenot R., Robert F., Dorémieux-Morin C., Chottard G., Ledon H., Jeannin Y., Brégeault J.-M. ${ }^{31} \mathrm{P}$ and ${ }^{183} \mathrm{~W}$ NMR spectroscopic evidence for novel peroxo species in the " $\mathrm{H}_{3}\left[\mathrm{PW}_{12} \mathrm{O}_{40}\right] \cdot \mathrm{yH}_{2} \mathrm{O} / \mathrm{H}_{2} \mathrm{O}_{2}$ " system. Synthesis and X-ray structure of tetrabutylammonium ( $\mu$-hydrogen phosphate)bis( $\mu$-peroxo)bis(oxoperoxotungstate)(2-): a catalyst of olefin epoxidation in a biphase medium. Inorg. Chem. 1994. Vol. 33(5), P. 871-878.

7. Kuznetsova L.I., Kuznetsova N.I., Maksimovskaya R.I., Koscheeva O.S., Utkin V.A. Catalytic properties of heteropoly compounds in 1,3-butadiene oxidation with hydrogen peroxide. Kinetics and Catalysis 2013. Vol. 54(4), P. 420-430.

8. Кузнецова Н.И., Детушева Л.Г., Кузнецова Л.И., Федотов М.А., Лихолобов В.А. Катализ гетерополикомплексами реакций окисления циклогексена и разложения пероксида водорода. Кинетика и катализ 1992. Т. 33(3), C. 516-523. [Kuznetsova N.I., Detusheva L.G., Kuznetsova L.I., Likholobov V.A. Oxidation of cyclohexene and decay of hydrogen peroxide catalyzed by heteropolycomplexes. Kinetics and Catalysis 1992. Vol. 33(3), P. 516-523 (In Russ.)].

9. Maksimovskaya R.I., Maksimov G.M. ${ }^{31} \mathrm{P}$ NMR studies of hydrolytic conversion of 12-tungstophosphoric heteropolyacid. Coord. Chem. Rev. 2019. 385, P. 81-89.

10. Fuchs J. Thiele A., Palm R. Z. Struktur und schwingungsspektrum des

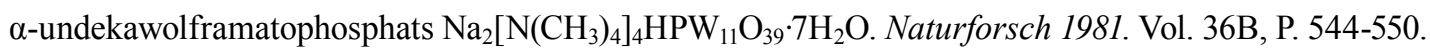

11. Kuznetsova L.I., Kuznetsova N.I., Koscheeva O.S. Promoting effect of ionic liquids in liquidphase oxidation of cyclohexane with a mixture of $\mathrm{O}_{2}$ and $\mathrm{H}_{2}$. Catal. Commun. 2017. Vol. 88, P. 50-52.

12. Koscheeva O.S., Kuznetsova N.I., Kuznetsova L.I. Quantification of small amounts of ionic liquids in solutions using CHN analysis. Rus. Chem. Bull., Intern. Edition 2018. Vol. 67(9), P. 1617-1620.

13. Choundhary V.R., Samanta C. Role of chloride or bromide anions and protons for promoting the selective oxidation of $\mathrm{H}_{2}$ by $\mathrm{O}_{2}$ to $\mathrm{H}_{2} \mathrm{O}_{2}$ over supported Pd catalysts in an aqueous medium. $J$. Catal. 2006. Vol. 238, P. 28-38.

14. Maksimovskaya R.I. Molybdophosphate heteropoly blues: electron-transfer reactions in aqueous solutions as stydied by NMR. Polyhedron 2013. Vol. 65, P. 54-59.

15. Kuznetsova L.I., Kuznetsova N.I. Cyclohexane oxidation with an $\mathrm{O}_{2}-\mathrm{H}_{2}$ mixture in the presence of a two-component $\mathrm{Pt} / \mathrm{C}$ - heteropoly acid catalyst and ionic liquids. Kinetics and Catalysis 2017. Vol. 58(5), P. 522-532.

16. Abhabarari B., Dorostkar N., Martinez-Huerta M.N. Synthesis of biodiesel from Nigella sativa oil using surfactant-Brønsted acidic-combined ionic liquid as catalyst. Fuel Proc. Techn. 2014. Vol. 118, P. 296-301. 
17. Hawkins C.A., Rud A., Guthric M.L., Dietz M.L. Rapid quantification of imidazolium-bazed ionic liquids by hydrophilic interaction liquid chromatography: Metodology and an investigation of the retention mechanisms. J. Chromatogr. A 2015. Vol. 1400, 54-64.

18. Markovska A., Stepnovski P. Capillary isotachophoresis for the analysis of ionic liquids entities. J. Sep. Sci. 2010. Vol. 33, P. 1991-1996. 